\title{
MICROSATELLITE MARKERS BASED ASSESSMENT OF RUST RESISTANCE GENES IN PAKISTANI BREAD WHEAT GENOTYPES
}

\author{
N. Akhtar ${ }^{*}$, A. Kiran ${ }^{1}$, Sajid-ur-Rehman², M. Kausar ${ }^{1}$, M. Khan $^{1}$, U. Saleem ${ }^{1}$ and T. Mahmood ${ }^{3}$ \\ ${ }^{1}$ Department of Plant Breeding and Genetics, College of Agriculture, University of Sargodha, Sargodha, 40100, Pakistan \\ ${ }^{2}$ Agriculture Biotechnology Research Institute, Ayub Agriculture Research Institute, Faisalabad, 38000, Pakistan \\ ${ }^{3}$ Department of Plant Breeding and Genetics, PMAS Arid Agriculture University, Rawalpindi, 46000, Pakistan \\ *Corresponding Author’s email: naeem.siraj@uos.edu.pk, naeem.uca@gmail.com
}

\begin{abstract}
Development of rust resistant wheat cultivars to meet ever-increasing wheat production targets for national food security in climate change scenario is currently at high priority in Pakistan. Focusing on this, here we report the screening of 40 wheat genotypes including advanced breeding lines and commercial varieties through PCR based DNA markers to detect genes conferring resistance against different rust races. In this study $22 \mathrm{SSR} / \mathrm{STS}$ markers were used which included nine leaf rust (Lr19, Lr22a, Lr27, Lr28, Lr34, Lr35, Lr37, Lr46 and Lr67), eight stripe rust (Yr5, Yr15, Yr17, Yr18, Yr26, Yr29, Yr30 and Yr46) and seven stem rust resistance genes ( $\mathrm{Sr} 2, \mathrm{Sr} 22, \mathrm{Sr} 24, \mathrm{Sr} 25, \mathrm{Sr} 26, \mathrm{Sr} 38$ and $\mathrm{Sr} 39)$. The results revealed that allelic frequency for rust resistance genes ( $\mathrm{Lr}, \mathrm{Yr}$ and $\mathrm{Sr}$ ) was maximum (70.4\%) in three advanced wheat lines namely V-9452, V11153 and NNG-3. However, minimum frequency (51.9\%) was found in 25 genotypes. Three advanced lines NNG-3, V9432 and V-11153 had maximum number of rust resistance genes among all the reported wheat genotypes which indicated narrow genetic base of Pakistani wheat cultivars for rust resistance. Therefore, it is suggested that newly discovered rust resistance genes especially Lr and Yr should be used along with existing genes for effective and durable rust resistance in new wheat varieties.
\end{abstract}

Key words: Gene, heat map, DNA markers, PCR, SSR.

\section{INTRODUCTION}

Wheat (Triticum aestivum L.) is widely grown and consumed food crop worldwide. It gives about $20 \%$ of calories consumed by the world population. Its importance is increasing with increasing global population (Tadesse et al., 2013; Akhtar et al., 2018). It is also an important cereal crop of Pakistan due to its consumption as staple food of more than 220 million populations. In Pakistan, bread wheat is cultivated on 8740 thousand hectares having production of 25.195 million tons (Government of Pakistan, 2019). Although Pakistan has attained selfsufficiency in wheat production but per hectare yield is still lower than other wheat producing countries (FAO, 2018). Therefore, advanced technologies need to be adopted to save wheat crop from biotic and abiotic stresses and to enhance wheat production to fulfill the food requirements of large population.

It is observed that in Pakistan rusts reduce wheat yield nearly $10 \%$ every year in later growth stages. But in early growth stages, yield losses may have reached up to 20\% (Ejaz et al., 2012; Singh et al., 2016). Wheat rusts caused by Puccinia spp. are among the major production constraints worldwide (Qamar et al., 2014; Bhardwaj et al., 2019). Leaf rust (Lr) attack on wheat occurs more frequently than stripe rust (Yr) or stem rust (Sr). Pakistan suffered from leaf rust epidemics in 1972-73 when the disease severity level was $30-50 \%$ while in 1976-78, disease severity increased up to $50-80 \%$ (Khan, 1997). Similarly, in 1995, 2005 and 2019 stripe rust and leaf rust epidemics have caused significant yield losses (Qamar et al., 2014 and Gessese, 2019). The emergence of new races of stem rust ( $\mathrm{Sr}$ ) has brought a major challenge of world wheat production (Gessese, 2019) The prime objective of wheat breeders is to develop new wheat varieties with optimum levels of disease resistance (McCallum et al., 2016). The wheat germplasm has a large set of resistance genes and more than 50 wheat rust resistant genes for each of the three rust species have been reported by different wheat researchers (Pretorius et al., 2012; Pal et al., 2015; Gessese, 2019).

Previously, conventional breeding techniques were used for development of new rust resistant wheat genotypes through gene recombination but recently, marker assisted selection, by far, the most efficient and broadly used tool for the monitoring of Pst pathotypes and threats of Ug99-complex races (Bhardwaj et al., 2019; Gessese, 2019). Microsatellites (SSR/STS) as reported in the literature are one of the most suitable molecular markers for the detection of rust resistance genes in hexaploid wheat (Singh et al., 2016). At present, approximately 2,800 SSRs have been reported and genetically mapped in wheat genome to investigate wheat rust resistant genes. 
This study was designed to identify rust resistant genes in candidate wheat varieties and existing commercial cultivars by using DNA based markers tightly linked to rust resistance genes in bread wheat.

\section{MATERIALS AND METHODS}

Healthy seeds of 40 wheat genotypes (30 advanced lines and 10 commercial varieties) were sown in the experimental area of Agricultural Biotechnology Research Institute (ABRI), Ayub Agriculture Research Institute, Faisalabad, Pakistan in Alpha-Lattice design with two replications (Table 1). At tillering stage, 2-3 fresh leaves of selected plants from each genotype were collected and stored at $-20^{\circ} \mathrm{C}$. A modified CTAB method was used to extract DNA of studied wheat genotypes (Rahman et al., 2002).

The quality of extracted DNA was analyzed by gel electrophoresis using $0.8 \%$ agarose gel. The concentration of total genomic DNA of 40 wheat lines/varieties was determined by an automated NanoDrop-1000 3.3.1 apparatus. Quantified DNA was diluted to the working concentration of about $30 \mathrm{ng} / \mu 1$.

A total of 22 Simple Sequence Repeats (SSR) and Simple Tendom Sequence (STS) markers were used in the experiment to identify genes that conferred resistance to various rust races in wheat genotypes (Table 2) along with 20 gene differentials to confirm the presence of resistant gene of interest in studied wheat genotypes. DNA amplification was done in a thermo-cycler machine using volume of $20 \mu \mathrm{l}$ per sample having $2 \mu \mathrm{l} 10 \mathrm{X}$ Taq buffer, $2 \mu \mathrm{l}$ $\mathrm{MgCl}_{2}(25 \mathrm{mM}), 1 \mu \mathrm{l}$ each of dATP, dCTP, dTTP and dGTP $(2.5 \mathrm{mM}), 1.5 \mu \mathrm{l}$ primer $(30 \mathrm{ng} / \mu \mathrm{l}), 2 \mu \mathrm{l}$ of genomic DNA (30 ng/ $\mu \mathrm{l}), 0.2 \mu \mathrm{l}$ (5unit/ $\mathrm{ll}$ ) Taq DNA Polymerase and 8.3 $\mu \mathrm{ldd} \mathrm{H}_{2} \mathrm{O}$.

Amplification reactions were programmed for 40 cycles. Amplification products were electrophoresed in $3 \mathrm{~g} / \mathrm{l}$ agarose gel. DNA ladder of $1000 \mathrm{bp}$ with known molecular weight bands was loaded along with PCR amplified DNA products on gel to determine the size/molecular weight of the polymorphic DNA fragments. Then amplified products were photographed using Syngene Gel Documentation System. Polyacrylamide Gel Electrophoresis (PAGE) was also performed for those samples which had small DNA fragments to study them precisely. Presence of rust resistant gene with adequate band size was scored as positive $(+)$ while susceptible gene was scored as negative (-) as shown in Tables $6,7 \& 8$.

Estimation of allelic frequency (\%) and allelic diversity were determined by R. and Power Maker V.0.3 software package. The polygenetic analysis with reference to rust resistant genes was determined by using R-software version 3.0.2. All amplified loci based on SSR/STS banding pattern were recorded as present (1) and absent (0) in the 40 genotypes. The bivariate 1-0 was used to construct heat map dendrogram by using cluster method between group linkage and genetic distance was determined through Squared Euclidean Distance.

\section{RESULTS}

A total of 22 molecular markers (SSR/STS) were used in this study to determine the presence/absence of rust resistance genes $(\mathrm{Lr}, \mathrm{Yr} \& \mathrm{Sr})$ in 40 wheat genotypes (Table 2). Xgwm533, Xwmc44, Xgwm11, Lr28, csLV34, Sr26\#43, BF145935, Xcfd23, Xcfd71, Xbarc352, cfa2123, SCS253 and Xgwm296 provided reproducible results whereas csSr2, Sr39\#22r, Sr39F 2 , STS7/8, STS9/10, BARC71, Xgwm437, Ventriup LN2 and SCS265 failed to amplify fragments for Sr24, Lr37 and Yr5 due to some experimental error. Gene differentials (20) were also used to confirm band size of rust resistant genes in studied wheat genotypes. Regarding leaf rust (Lr) resistance genes, nine genes were investigated. Among these, five leaf rust (Lr) resistance genes Lr.19. Lr.22a, Lr.28, Lr.35 and Lr.67 genes were identified in all studied wheat genotypes while Lr.24 and Lr.37 were not found. Whereas, Lr.34 and Lr.46 genes were present in 10-B9346 and NR-310 wheat genotypes, respectively (Table 3). Regarding eight studied stripe rust resistance conferring genes, only Yr.15, Yr.26 Yr.30 and Yr.46 were observed in all 40 wheat genotypes while Yr.5 and Yr.17 were absent in all genotypes. Stripe rust resistance genes Yr.18 and Yr. 29 were also identified in limited number of wheat genotypes i.e. 10B9346 and 10B2003 genotypes, respectively (Table 4). Stem rust resistance genes were examined in current wheat genotypes by using seven Sr. markers and it was observed that only Sr.2, Sr.22, Sr.25 and Sr.39 were present in all 40 wheat genotypes to confer stem rust resistance while Sr. 24, Sr.26 and Sr. 38 were lacking in all genotypes (Table 5). Phenotypic evaluation of wheat genotypes for leaf, stripe and stem rusts is presented in Table 9.

Allelic frequency of rust resistance genes ( $\mathrm{Lr}, \mathrm{Yr}$ and $\mathrm{Sr}$ ) was observed in studied wheat genotypes and found maximum frequency $(70.4 \%)$ in 3 advance lines namely $\mathrm{V}$ 9452, V-11153 and NNG-3. However minimum frequency $(51.9 \%)$ was estimated in twenty-five genotypes in which 10 were commercial varieties and other 15 were advanced wheat lines (Fig 1).

The genetic association among advanced wheat breeding lines for rust resistance genes was determined by developing heat map based on the SSR/STS banding patterns. Heat map indicated two groups; Group-A contained three clusters indicating wheat genotypes along $\mathrm{Y}$-axis, whereas, Group-B indicated clusters of rust resistance genes (along with their primers) on X-axis (Fig 2). Red colour in map indicated presence of rust resistance genes in respective genotypes. However, yellow colour indicated absence of required genes. Cluster-A comprised of two leaves. Leaf-1 contained two genes Sr25 and Lr19 while leaf-2 had 11 genes (Lr19, Lr22a, Lr28, Lr67, Yr49, Sr2, Yr30, Sr39, Sr22 and Yr15), which indicated the 
existence of rust resistance genes in 40 wheat genotypes. Cluster-B discriminated five rust resistance genes (Lr34, Lr46, Yr18, Yr29 and Sr57) in red and yellow patches and cluster-C indicated five genes (Yr5, Sr24, Lr37, Yr17 and $\mathrm{Sr} 38)$ in yellow patch for absence of resistance genes of rust. In group-B the cluster $\mathrm{A}^{\prime}$ comprised of two leaves. Leaf 1 contains three genotypes (NNG-3, V-9452 and V11153 ) with maximum allelic frequency of rust resistance genes $(70.4 \%)$, whereas leaf 2 contains eight genotypes (AARI-11, Ufaq-2002, TW86014, V-10355, 09B9110, V11161 , NR-310 and 10B2003) with $63 \%$ allelic frequency. Cluster B' comprised of two leaves 4 and 5. Leaf 4 contained the largest number of 24 genotypes with allelic frequency of $51.9 \%$. However, leaf 5 contains three genotypes (NR-378, V-11156 and TW96018) showing $59.3 \%$ allelic frequency. Cluster $C^{\prime}$ comprised of Leaf 3 contains only one genotype (Seher-06) with $59.3 \%$ allelic frequency.
All this characterization of leaf rust, stripe rust and stem rust resistant genes represented by + and - signs in wheat genotypes showed in tables 6,7 and 8, respectively.

Sr.25 is one of the stem rust resistance gene which exhibits strong linkage with Lr.19 in wheat for adult plant resistance. In this study, the primer BF145935 amplified $198 \mathrm{bp}$ and 180bp DNA fragments of Sr.25 gene in all the studied wheat genotypes which conferred stem rust resistance at two fragment lengths (Fig 3). DNA fragment length of $330 \mathrm{bp}$ linked with primer Lr28 indicated the presence of leaf rust resistance gene Lr.28 in all studied wheat genotypes (Fig 4). The primer Xgwm11 amplified 213 bp DNA fragment length for Yr. 15 gene in most of the studied wheat genotypes which indicated the occurrence of stripe rust resistance gene. The banding pattern on agarose gel only for 10 wheat genotypes. The results of remaining gels are not reported here (Fig 5).

Table 1. Name of wheat varieties and advanced breeding lines as supplementary material.

\begin{tabular}{llllll}
\hline $\begin{array}{l}\text { S. } \\
\text { No. }\end{array}$ & $\begin{array}{l}\text { Name of wheat } \\
\text { genotypes }\end{array}$ & Source & S. No. & $\begin{array}{l}\text { Name of wheat } \\
\text { genotypes }\end{array}$ & Source \\
\hline 1. & NR-399 & NARC, Islamabad & 21. & V-10031 & WRI Faisalabad \\
2. & V-9452 & UAF Faisalabad & 22. & Shafaq-06 & WRI Faisalabad \\
3. & V-11153 & WRI Faisalabad & 23. & SH-2002 & WRI Faisalabad \\
4. & V-11154 & WRI Faisalabad & 24. & V-10104 & WRI Faisalabad \\
5. & NNG-3 & NIBGE Faisalabad & 25. & TW96018 & AZRI Bhakkar \\
6. & V-11156 & WRI Faisalabad & 26. & V-10110 & WRI Faisalabad \\
7. & NR-310 & NARC, Faisalabad & 27. & V-11166 & WRI Faisalabad \\
8. & V-11160 & WRI Faisalabad & 28. & NR-400 & NARC, Islamabad \\
9. & V-10B2003 & RARI Bahwalpur & 29. & V-TW86014 & AZRI Bhakkar \\
10. & V-11161 & WRI Faisalabad & 30. & NR-399 & NARC, Islamabad \\
11. & V-10-B9346 & RARI Bahwalpur & 31. & NR-378 & NARC, Islamabad \\
12. & V-11164 & WRI Faisalabad & 32. & Pb-11 & WRI Faisalabad \\
13. & V-09B9110 & RARI Bahwalpur & 33. & Galaxy-13 & WRI Faisalabad \\
14. & V-10355 & WRI Faisalabad & 34. & Ufaq-2002 & WRI Faisalabad \\
15. & V-09-BT043 & ABRI Faisalabad & 35. & AARI-11 & WRI Faisalabad \\
16. & V-10217 & WRI Faisalabad & 36. & Sehar-06 & WRI Faisalabad \\
17. & V-10-BT002 & ABRI Faisalabad & 37. & Inq-91 & WRI Faisalabad \\
18. & V-09B9172 & RARI Bahwalpur & 38. & Millat-11 & WRI Faisalabad \\
19. & V-10025 & WRI Faisalabad & 39. & Fsd-08 & WRI Faisalabad \\
20. & V-10193 & WRI Faisalabad & 40. & Lasani-08 & WRI Faisalabad \\
\hline
\end{tabular}

Table 2. List of SSR/STS markers demonstrating in the experiment.

\begin{tabular}{llllll}
\hline Sr.\# & Primer Name & Linked gene & Location & Size (bp) & Reference \\
\hline 1 & Xgwm 296 & Lr22a & 2DS & $121 / 131$ & Hiebert et al., 2007 \\
2 & Xwmc 44 & Lr46/Yr29 & 1BL & 242 & Suenaga et al. $(2001)$ \\
3 & Xgwm 437 & Lr19 & 7DL & 130 & (http://maswheat.ucdavis.edu) \\
4 & X-barc 352 & Lr34/Yr18 & 4D.7DS & 250 & (http://maswheat.ucdavis.edu) \\
5 & Xgwm-11 & Yr15/Yr26 & 1B,1BS & 213 & (http://maswheat.ucdavis.edu) \\
6 & Xgwm-533 & Sr2 & 3B & 120 & Spielmeyer et al., (2003) \\
7 & Cfa2123 & Sr22 & 7A & $245 / 260$ & Hiebert et al., 2010 \\
8 & csSr2 & Sr2/Yr30 & 3B & 378 & (http://maswheat.ucdavis.edu) \\
9 & Lr28 & Lr28 & 4AL & 330 & (http://www.graingenes.com)
\end{tabular}




\begin{tabular}{llllll}
10 & BF145935 & Sr25/Lr19 & 7D.7A & $180 / 198$ & $\begin{array}{l}\text { Mago } \text { et al., 2011 } \\
\text { Campbell } \text { et al., 2009 }\end{array}$ \\
11 & STS(7/8) & Yr5 & 1B & 500 & Liu et al., 2010 \\
12 & Sr26\#43 & Sr26 & 6AS.6AL & 206 & (http://maswheat.ucdavis.edu) \\
13 & csLv34 & Lr34/Yr18 & 7DS & 150 & Mago et al., 2011 \\
14 & Sr39\#22r & Sr39/Lr35 & 2B & 487 & Hiebert et al., 2010 \\
15 & Xcfd71 & Lr67/Yr26 & 4DL & 214 & Hiebert et al., 2010 \\
16 & Xcfd23 & Lr67 & 4DL & 211 & (http://maswheat.ucdavis.edu) \\
17 & BARC71 & Sr24/Lr24 & 3DL & $85 / 103$ & (http://maswheat.ucdavis.edu) \\
18 & STS-9/10 & Yr5/Yr17 & 2BL & 439 & (http://maswheat.ucdavis.edu) \\
19 & SCS253 & Lr19 & 7D.7A & 736 & (http://maswheat.ucdavis.edu) \\
20 & Ventriup LN2 & Lr37/Sr38 & 2AS & 259 & (http://maswheat.ucdavis.edu) \\
21 & SCS265 & Lr19 & 7D.7Ag & 512 & (http://maswheat.ucdavis.edu) \\
22 & Sr39F 2 & Sr39 & 2BS & 900 & \\
\hline
\end{tabular}

Table 3. Wheat genotypes showing presence/absence of leaf rust (Lr) resistance of genes.

\begin{tabular}{|c|c|c|c|}
\hline Sr.\# & Primers & Wheat genotypes & Lr genes \\
\hline 1 & SCS265 & All genotypes were positive for this marker. & $(\operatorname{Lr} 19)+\mathrm{ve}$ \\
\hline 2 & Xgwm 296 & All genotypes were positive for this marker. & $(\operatorname{Lr} 22 a)+v e$ \\
\hline 3 & BARC71 & All genotypes were negative for this marker. & (Lr24) -ve \\
\hline 4 & Lr28 & All genotypes were positive for this marker. & $(\operatorname{Lr} 28)+v e$ \\
\hline 5 & Xbarc-352Lr34 & $\begin{array}{l}\text { 9452, 11153, NNG-3, NR-310, 10B2003, 11161, 09B9110, } 10355 \text {, } \\
\text { TW86014, Ufaq-2002, AARI-11 were positive for this marker. }\end{array}$ & $(\operatorname{Lr} 34)+v e$ \\
\hline 6 & Sr39\#22r & All genotypes were positive for this marker. & $(\mathrm{Lr} 35)+\mathrm{ve}$ \\
\hline 7 & VentriupLN2 & All genotypes were negative for this marker. & $(\mathrm{Lr} 37)$-ve \\
\hline 8 & Xwmc-44 & $\begin{array}{l}9452,11153, \text { NNG-3, NR-378, 11156, TW96018, Sehar-06 were positive } \\
\text { for this marker. }\end{array}$ & $(\mathrm{Lr} 46)+\mathrm{ve}$ \\
\hline 9 & $\operatorname{Xcfd} 23$ & All genotypes were positive for this marker. & $($ Lr67)+ve \\
\hline
\end{tabular}

Table 4. Wheat genotypes showing presence/absence of stripe rust (Yr) resistance genes.

\begin{tabular}{|c|c|c|c|}
\hline Sr.\# & Primers & Wheat genotypes & Yr genes \\
\hline 1 & $\operatorname{STS}(7-8)$ & All genotypes were negative for this marker. & $($ Yr5) -ve \\
\hline 2 & Xgwm-11 & All genotypes were positive for this marker. & $(\mathrm{Yr} 15)+\mathrm{ve}$ \\
\hline 3 & STS9/10 & All genotypes were negative for this marker. & $(Y r 17)-v e$ \\
\hline 4 & $\operatorname{csLv} 34$ & $\begin{array}{l}\text { 9452, 11153, NNG-3, NR-310, 10B2003, 10355, 11161, TW86014, } \\
\text { Ufaq-2002, AARI-11,09B9110 were positive for this marker. }\end{array}$ & $($ Yr18)+ve \\
\hline 5 & Xgwm-11 & All genotypes were positive for this marker. & $(Y r 26)+v e$ \\
\hline 6 & Xwmc-44 & $\begin{array}{l}\text { 9452, } 11153 \text {, NNG-3, NR-310, 11156, , 10355, TW96018, NR-378, } \\
\text { Sehar-06 }\end{array}$ & $($ Yr29) +ve \\
\hline 7 & Xgwm-533 & All genotypes were positive for this marker. & $($ Yr30) +ve \\
\hline 8 & Xcfd71 & All genotypes were positive for this marker. & $(Y r 46)+v e$ \\
\hline
\end{tabular}

Table 5. Wheat genotypes showing presence/absence of stem rust (Sr) resistance genes.

\begin{tabular}{|c|c|c|c|}
\hline Sr.\# & Primers & Wheat genotypes & Sr genes \\
\hline 1 & Xgwm-533 & All genotypes were positive for this marker. & $(\mathrm{Sr} 2)+\mathrm{ve}$ \\
\hline 2 & $\mathrm{cfa} 2123$ & All genotypes were positive for this marker. & $(\mathrm{Sr} 22)+\mathrm{ve}$ \\
\hline 3 & $\operatorname{Sr} 24$ & All genotypes were negative for this marker. & $(\mathrm{Sr} 24)-\mathrm{ve}$ \\
\hline 4 & BF145935 & All genotypes were positive for this marker. & $(\mathrm{Sr} 25)+\mathrm{ve}$ \\
\hline 5 & Sr26\#43 & All genotypes were negative for this marker. & $(\mathrm{Sr} 26)-\mathrm{ve}$ \\
\hline 6 & Ventriup/LN2 & All genotypes were negative for this marker. & $(\mathrm{Sr} 38)-\mathrm{ve}$ \\
\hline 7 & Sr39\#22r & All genotypes were positive for this marker. & $(\mathrm{Sr} 39)+\mathrm{ve}$ \\
\hline
\end{tabular}


Table 6. Characterization of Leaf Rust Resistant Genes (Lr) in Wheat Genotypes.

\begin{tabular}{|c|c|c|c|c|c|c|c|c|c|c|}
\hline Sr.\# & Genotypes & Lr19 & Lr22a & Lr24 & Lr28 & Lr34 & Lr35 & Lr37 & Lr46 & Lr67 \\
\hline 1. & NR-399 & + & + & - & + & - & + & - & - & + \\
\hline 2. & V-9452 & + & + & - & + & + & + & - & + & + \\
\hline 3. & V-11153 & + & + & - & + & + & + & - & + & + \\
\hline 4. & V-11154 & + & + & - & + & - & + & - & - & + \\
\hline 5. & NNG-3 & + & + & - & + & + & + & - & + & + \\
\hline 6. & V-11156 & + & + & - & + & - & + & - & + & + \\
\hline 7. & NR-310 & + & + & - & + & + & + & - & - & + \\
\hline 8. & V-11160 & + & + & - & + & - & + & - & - & + \\
\hline 9. & 10B2003 & + & + & - & + & + & + & - & - & + \\
\hline 10. & V-11161 & + & + & - & + & + & + & - & - & + \\
\hline 11. & 10-B9346 & + & + & - & + & - & + & - & - & + \\
\hline 12. & V-11164 & + & + & - & + & - & + & - & - & + \\
\hline 13. & 09B9110 & + & + & - & + & + & + & - & - & + \\
\hline 14. & V-10355 & + & + & - & + & + & + & - & - & + \\
\hline 15. & 09-ВТ043 & + & + & - & + & - & + & - & - & + \\
\hline 16. & V-10217 & + & + & - & + & - & + & - & - & + \\
\hline 17. & 10-ВТ002 & + & + & - & + & - & + & - & - & + \\
\hline 18. & 09B9172 & + & + & - & + & - & + & - & - & + \\
\hline 19. & V-10025 & + & + & - & + & - & + & - & - & + \\
\hline 20. & V-10193 & + & + & - & + & - & + & - & - & + \\
\hline 21. & V-10031 & + & + & - & + & - & + & - & - & + \\
\hline 22. & Shafaq-06 & + & + & - & + & - & + & - & - & + \\
\hline 23. & SH-2002 & + & + & - & + & - & + & - & - & + \\
\hline 24. & V-10104 & + & + & - & + & - & + & - & - & + \\
\hline 25. & TW96018 & + & + & - & + & - & + & - & + & + \\
\hline 26. & V-10110 & + & + & - & + & - & + & - & - & + \\
\hline 27. & V-11166 & + & + & - & + & - & + & - & - & + \\
\hline 28. & NR-400 & + & + & - & + & - & + & - & - & + \\
\hline 29. & TW86014 & + & + & - & + & + & + & - & - & + \\
\hline 30. & KANZO & + & + & - & + & - & + & - & - & + \\
\hline 31. & NR-378 & + & + & - & + & - & + & - & + & + \\
\hline 32. & $\mathrm{~Pb}-11$ & + & + & - & + & - & + & - & - & + \\
\hline 33. & V-07096 & + & + & - & + & - & + & - & - & + \\
\hline 34. & Ufaq-2002 & + & + & - & + & + & + & - & - & + \\
\hline 35. & AARI-11 & + & + & - & + & + & + & - & - & + \\
\hline 36. & Seher-06 & + & + & - & + & - & + & - & + & + \\
\hline 37. & Inq-91 & + & + & - & + & - & + & - & - & + \\
\hline 38. & Millat-11 & + & + & - & + & - & + & - & - & + \\
\hline 39. & Fsd-08 & + & + & - & + & - & + & - & - & + \\
\hline 40. & Lasani-08 & + & + & - & + & - & + & - & - & + \\
\hline
\end{tabular}

Table 7. Characterization of Stripe Rust Resistant Genes (Yr) in Wheat Genotypes.

\begin{tabular}{llcccccccc}
\hline Sr.\# & Genotypes & Yr5 & Yr15 & Yr17 & Yr18 & Yr26 & Yr29 & Yr30 & Yr46 \\
\hline 1. & NR-399 & - & + & - & - & + & - & + & + \\
2. & V-9452 & - & + & - & + & + & + & + & + \\
3. & V-11153 & - & + & - & + & + & + & + & + \\
4. & V-11154 & - & + & - & - & + & - & + & + \\
5. & NNG-3 & - & + & - & + & + & + & + & + \\
6. & V-11156 & - & + & - & - & + & + & + & + \\
7. & NR-310 & - & + & - & + & + & - & + & + \\
8. & V-11160 & - & + & - & - & + & - & + & +
\end{tabular}




\begin{tabular}{|c|c|c|c|c|c|c|c|c|c|}
\hline 9. & 10B2003 & - & + & - & + & + & - & + & + \\
\hline 10. & V-11161 & - & + & - & + & + & - & + & + \\
\hline 11. & 10-B9346 & - & + & - & - & + & - & + & + \\
\hline 12. & V-11164 & - & + & - & - & + & - & + & + \\
\hline 13. & 09B9110 & - & + & - & + & + & - & + & + \\
\hline 14. & V-10355 & - & + & - & + & + & - & + & + \\
\hline 15. & 09-ВT043 & - & + & - & - & + & - & + & + \\
\hline 16. & V-10217 & - & + & - & - & + & - & + & + \\
\hline 17. & 10-BT002 & - & + & - & - & + & - & + & + \\
\hline 18. & 09B9172 & - & + & - & - & + & - & + & + \\
\hline 19. & V-10025 & - & + & - & - & + & - & + & + \\
\hline 20. & V-10193 & - & + & - & - & + & - & + & + \\
\hline 21. & V-10031 & - & + & - & - & + & - & + & + \\
\hline 22. & Shafaq-06 & - & + & - & - & + & - & + & + \\
\hline 23. & SH-2002 & - & + & - & - & + & - & + & + \\
\hline 24. & V-10104 & - & + & - & - & + & - & + & + \\
\hline 25. & TW96018 & - & + & - & - & + & + & + & + \\
\hline 26. & V-10110 & - & + & - & - & + & - & + & + \\
\hline 27. & V-11166 & - & + & - & - & + & - & + & + \\
\hline 28. & NR-400 & - & + & - & - & + & - & + & + \\
\hline 29. & TW86014 & - & + & - & + & + & - & + & + \\
\hline 30. & KANZO & - & + & - & - & + & - & + & + \\
\hline 31. & NR-378 & - & + & - & - & + & + & + & + \\
\hline 32. & $\mathrm{~Pb}-11$ & - & + & - & - & + & - & + & + \\
\hline 33. & V-07096 & - & + & - & - & + & - & + & + \\
\hline 34. & Ufaq-2002 & - & + & - & + & + & - & + & + \\
\hline 35. & AARI-11 & & + & - & + & + & - & + & + \\
\hline 36. & Seher-06 & - & + & - & - & + & + & + & + \\
\hline 37. & Inq-91 & - & + & - & - & + & - & + & + \\
\hline 38. & Millat-11 & - & + & - & - & + & - & + & + \\
\hline 39. & Fsd-08 & - & + & - & - & + & - & + & + \\
\hline 40. & Lasani-08 & - & + & - & - & + & - & + & + \\
\hline
\end{tabular}

Table 8. Characterization of Stem Rust Resistant Genes (Sr) in Wheat Genotypes.

\begin{tabular}{clccccccc}
\hline Sr.\# & Genotypes & Sr2 & Sr22 & Sr24 & Sr25 & Sr26 & Sr38 & Sr39 \\
\hline 1. & NR-399 & + & + & - & + & - & - & + \\
2. & V-9452 & + & + & - & + & - & - & + \\
3. & V-11153 & + & + & - & + & - & - & + \\
4. & V-11154 & + & + & - & + & - & - & + \\
5. & NNG-3 & + & + & - & + & - & - & + \\
6. & V-11156 & + & + & - & + & - & - & + \\
7. & NR-310 & + & + & - & + & - & - & + \\
8. & V-11160 & + & + & - & + & - & - & + \\
9. & $10 B 2003$ & + & + & - & + & - & - & + \\
10. & V-11161 & + & + & - & + & - & - & + \\
11. & $10-B 9346$ & + & + & - & - & - & - & + \\
12. & V-11164 & + & + & - & + & - & - & + \\
13. & $09 B 9110$ & + & + & - & + & - & - & + \\
14. & V-10355 & + & + & - & + & - & - & + \\
15. & $09-B T 043$ & + & + & - & + & - & - & + \\
16. & V-10217 & + & + & - & + & - & - & + \\
17. & $10-B T 002$ & + & + & - & + & - & - & + \\
18. & $09 B 9172$ & + & + & - & + & - & - & + \\
19. & V-10025 & + & + & - & + & - & - & +
\end{tabular}




\begin{tabular}{|c|c|c|c|c|c|c|c|c|}
\hline 20. & V-10193 & + & + & - & + & - & - & + \\
\hline 21. & V-10031 & + & + & - & + & - & - & + \\
\hline 22. & Shafaq-06 & + & + & - & + & - & - & + \\
\hline 23. & SH-2002 & + & + & - & + & - & - & + \\
\hline 24. & V-10104 & + & + & - & + & - & - & + \\
\hline 25. & TW96018 & + & + & - & + & - & - & + \\
\hline 26. & V-10110 & + & + & - & + & - & - & + \\
\hline 27. & V-11166 & + & + & - & + & - & - & + \\
\hline 28. & NR-400 & + & + & - & + & - & - & + \\
\hline 29. & TW86014 & + & + & - & + & - & - & + \\
\hline 30. & KANZO & + & + & - & + & - & - & + \\
\hline 31. & NR-378 & + & + & - & + & - & - & + \\
\hline 32. & Pb-11 & + & + & - & + & - & - & + \\
\hline 33. & V-07096 & + & + & - & + & - & - & + \\
\hline 34. & Ufaq-2002 & + & + & - & + & - & - & + \\
\hline 35. & AARI-11 & + & + & - & + & - & - & + \\
\hline 36. & Seher-06 & + & + & - & + & - & - & + \\
\hline 37. & Inq-91 & + & + & - & + & - & - & + \\
\hline 38. & Millat-11 & + & + & - & + & - & - & + \\
\hline 39. & Fsd-08 & + & + & - & + & - & - & + \\
\hline 40. & Lasani-08 & + & + & - & + & - & - & + \\
\hline
\end{tabular}

Table 9. Phenotypic expression of wheat genotypes for rust (Lr, Yr and Sr) resistance.

\begin{tabular}{|c|c|c|c|c|}
\hline Sr\# & Genotype & $\begin{array}{l}\text { Leaf Rust (Lr) Resistance } \\
\text { Genes }\end{array}$ & $\begin{array}{l}\text { Stripe Rust (Yr) } \\
\text { Resistance Genes }\end{array}$ & $\begin{array}{l}\text { Stem Rust (Sr) Resistance } \\
\text { Genes }\end{array}$ \\
\hline 1 & NR-399 & $\mathrm{MR}$ & MR & $\mathrm{MR}$ \\
\hline 2 & V-9452 & r $\mathrm{R}$ & $\mathrm{R}$ & $\mathrm{R}$ \\
\hline 3 & V-11153 & $\mathrm{R}$ & $\mathrm{R}$ & $\mathrm{R}$ \\
\hline 4 & 11154 & MR & MR & MR \\
\hline 5 & NNG-3 & $\mathrm{R}$ & $\mathrm{R}$ & $\mathrm{R}$ \\
\hline 6 & V-11156 & MR & MR & MR \\
\hline 7 & NR-310 & MS & MS & MR \\
\hline 8 & $V-11160$ & MS & MS & MS \\
\hline 9 & 10B2003 & MS & MS & MR \\
\hline 10 & V-11161 & MS & MS & MR \\
\hline 11 & $10-\mathrm{B} 9346$ & MS & MR & MR \\
\hline 12 & V-11164 & $\mathrm{S}$ & MS & MS \\
\hline 13 & 09B9110 & MS & MS & MR \\
\hline 14 & V-10355 & MS & MS & MR \\
\hline 15 & 09-ВТ043 & MS & MS & MR \\
\hline 16 & V-10217 & MS & MS & MR \\
\hline 17 & 10-BT002 & MS & MS & MR \\
\hline 18 & 09B9172 & $\mathrm{S}$ & MS & MR \\
\hline 19 & V-10025 & MS & MS & MR \\
\hline 20 & V-10193 & MS & MS & MR \\
\hline 21 & V-10031 & MS & MR & MS \\
\hline 22 & Shafaq-06 & MS & MS & MR \\
\hline 23 & SH-2002 & MS & MS & MR \\
\hline 24 & V-10104 & MS & MS & MR \\
\hline 25 & TW96018 & MS & MS & MR \\
\hline 26 & V-10110 & MS & MS & MR \\
\hline 27 & V-11166 & MS & MS & MR \\
\hline 28 & NR-400 & MS & MS & MR \\
\hline 29 & TW86014 & MS & MS & MR \\
\hline
\end{tabular}




\begin{tabular}{lllll}
30 & KANZO & MS & MS & MR \\
31 & NR-378 & MS & MS & MR \\
32 & Pb-11 & MS & MS & MR \\
33 & V07096 & MS & MS & MR \\
34 & Ufaq-02 & MS & MS & MR \\
35 & AARI-11 & MS & MS & MR \\
36 & Seher-06 & MS & MS & MR \\
37 & Inq-91 & MS & MS & MR \\
38 & Millet-11 & MS & MS \\
\hline 30 & Fsd-08 & MS & MS
\end{tabular}

Note: R: resistant, MR: moderately resistant, MS: moderately susceptible and S: susceptible.

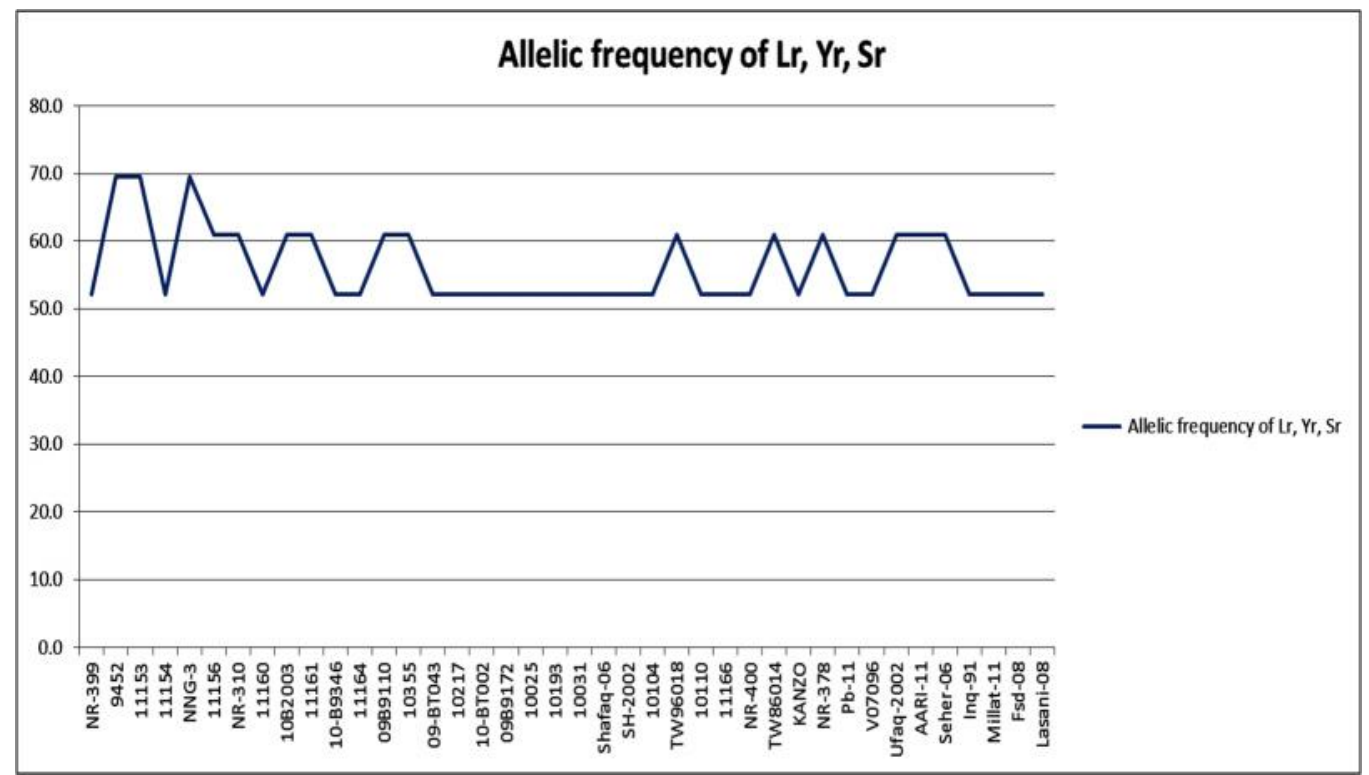

Figure 1. Allelic frequency of rust resistance genes in studied bread wheat genotypes.

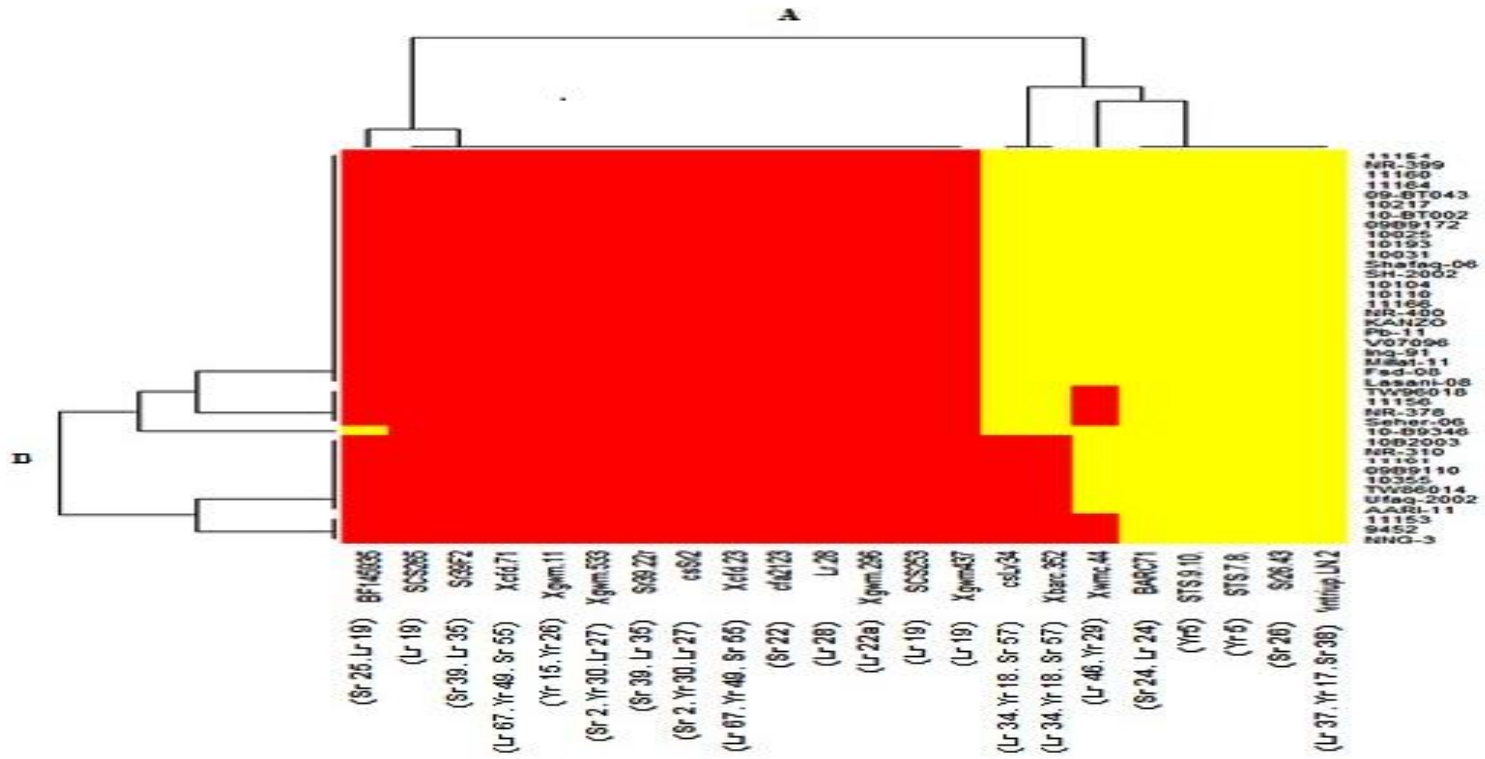

Figure 2. Heat map dandrogram showing genotype clusters along $\mathbf{Y}$-axis and gene clusters (with name of primer) along $\mathrm{X}$-axis. Red color in map indicated presence of rust resistance genes in respective genotypes. However, yellow color indicated absence of required genes. 


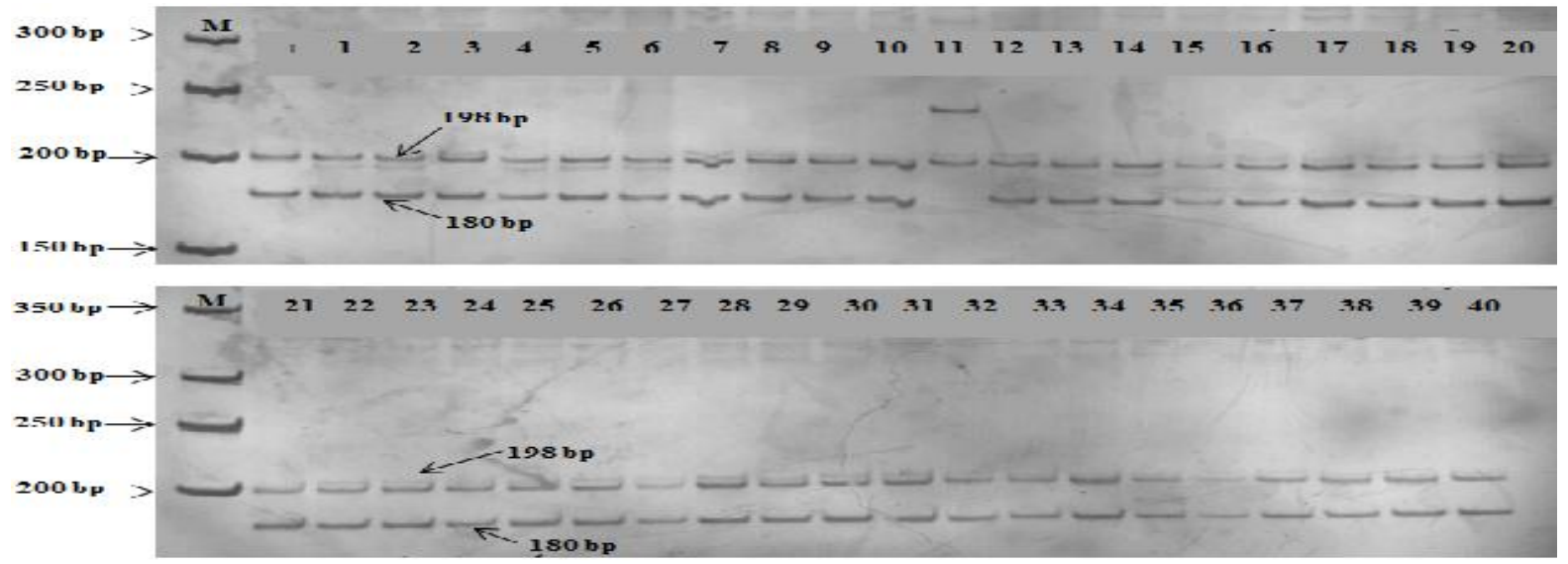

Figure 3. Wheat genotypes showing resistance against stem rust (Sr.25/Lr.19) by primer BF145935.

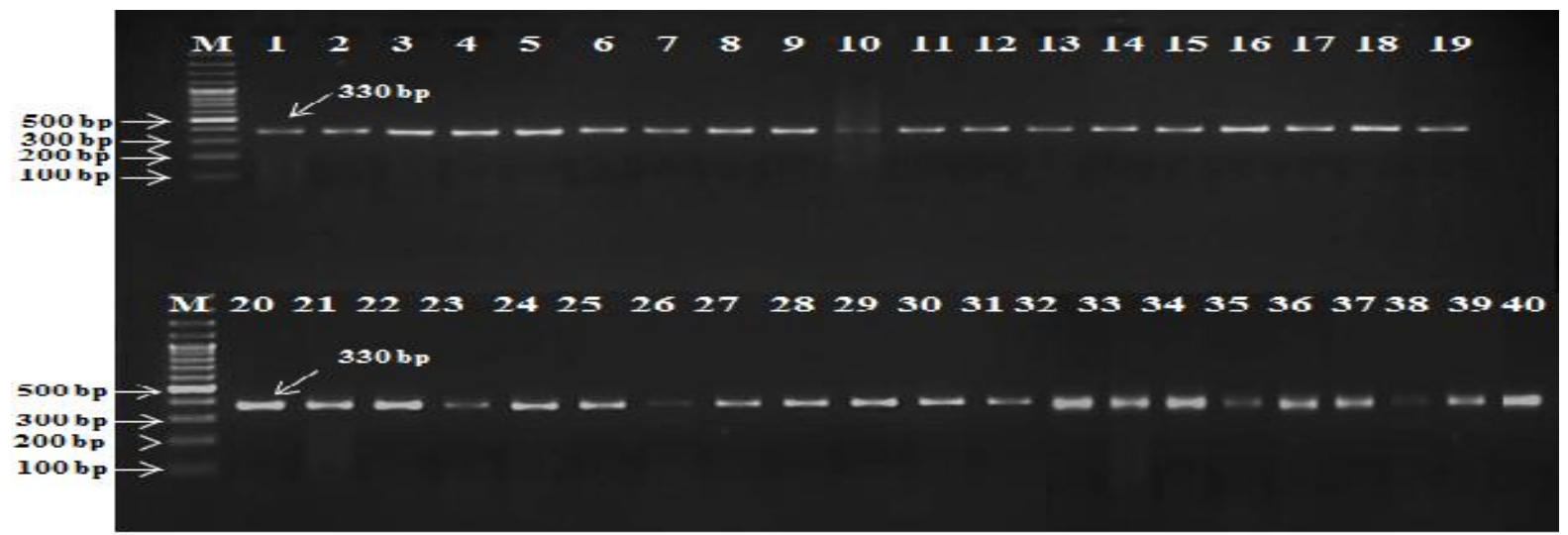

Figure 4. Wheat genotypes showing resistance against leaf rust (Lr.28) by primer Lr28.
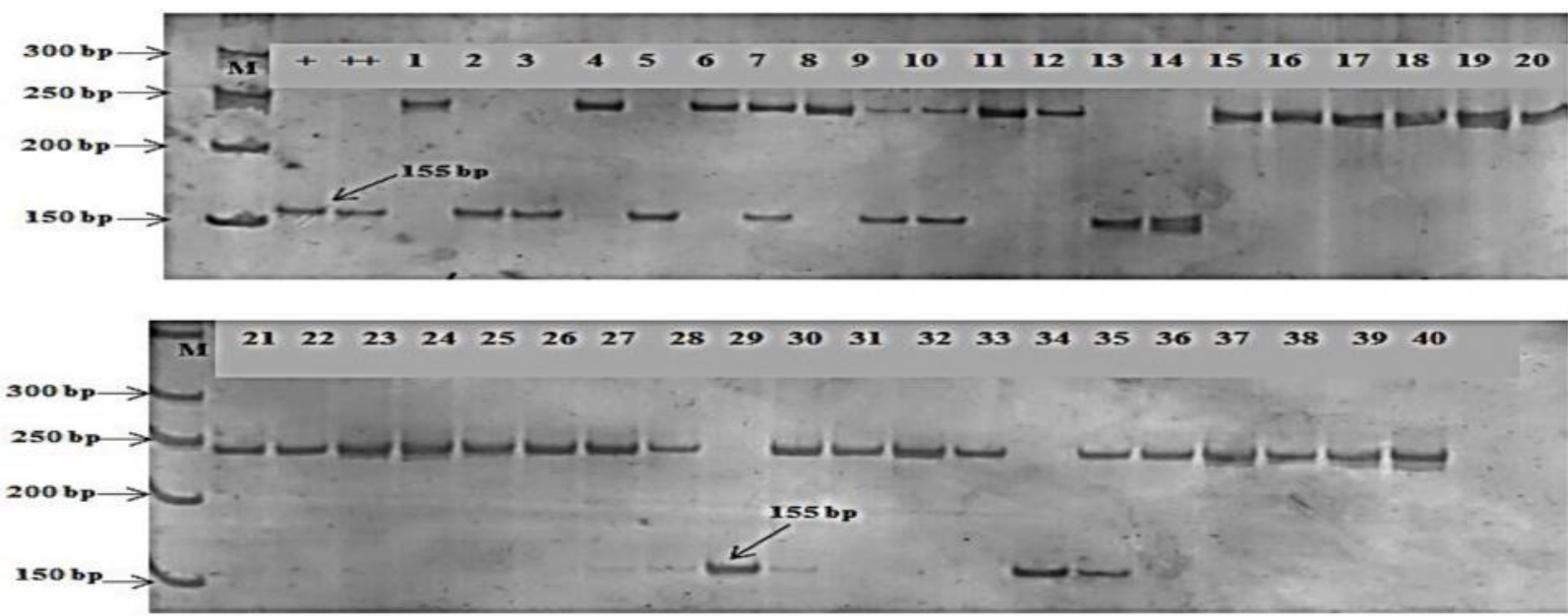

Figure 5. Wheat genotypes showing resistance against stripe rust (Yr. 18) by primer csLv34

\section{DISCUSSION}

The proposed study was aimed to screen advanced lines/commercial varieties of bread wheat developed by various wheat breeding institutes of Pakistan using molecular markers tightly linked with the genes conferring resistance to different rust races. Plant breeders broadly categorized rust resistance genes into seedling as well as adult plant resistance (APR) genes. The APR genes reveals durable, slow rusting, non-specific and horizontal resistance which are effective against rust epidemics (Jeffrey et al., 2014). Basnet et al., 2013 reported that at seedling stage rust resistance genes reveal the phenotypes of major effect and with varying infection types whereas 
most of the APR genes are limited in effect with varying disease severity levels. In this study various durable, slow rusting/non-specific rust resistance gene (Lr34/Yr18, Lr46/Yr29, Sr2/Yr30, Lr67/Yr49) have been observed. Based on the results, it was observed that only five out of nine reported Lr genes (Lr.19. Lr.22a, Lr.28, Lr.35 and Lr.67) were identified in all present wheat genotypes, while Lr.34 and Lr.46 genes were detected in 11 and 7 wheat genotypes respectively, which conferred leaf rust resistance in studied wheat genotypes. This indicated that a satisfactory number of leaf rust genes were not present in present Pakistani wheat genotypes and it was strongly suggested to broaden the wheat genetic back ground to cope with future rust epidemics (Qamar et al., 2014; McCallum et al., 2016, Bhardwaj et al., 2019; Gessese, 2019). Though in some cases, the results were not as per expectation as Lr. 24 did not show any band on agarose gel from the amplified DNA of studied wheat genotypes but previously these genes were observed in some bread wheat genotypes (Ejaz et al., 2012). This may be due to either improper amplification of PCR products by the primers or absence of these genes in studied wheat genotypes.

A total of eight stripe rust (Yr) resistance genes were observed and found four Yr resistance genes (Yr.15, Yr.26, Yr.30 \& Yr.46) in all studied wheat genotypes except Yr.5 and Yr.17 which did not show the bands on agarose gel. This might be the failure of STS primers to amplify the PCR products. Yr.18 and Yr.29 were also observed in different wheat genotypes. This indicated that Pakistani wheat genotypes have enough resistance against stripe rust pathogens. Our results get support from the results of Ejaz et al., (2012); Yu et al., (2012); Parveen et al., (2014); Wang et al., (2015); Bhardwaj et al., 2019).

Stem rust $(\mathrm{Sr})$ is an important disease of wheat throughout the world and resistance against $\mathrm{Sr}$ in newly developed wheat genotypes is very important to combat foreseen threat of Ug99 epidemics in Pakistan. A total of seven stem rust resistance genes ( $\mathrm{Sr} 2, \mathrm{Sr} 22, \mathrm{Sr} 24, \mathrm{Sr} 25$, Sr26, Sr38 and Sr39) were studied in 40 wheat genotypes using already reported primers which amplified the DNA fragments in wheat. Among these four stem rust genes (Sr2, Sr22, Sr25 and Sr39) were found in all studied wheat genotypes while the remaining did not show bands on agarose gel. It reveals that although Pakistani wheat genotypes have enough resistance against stem rust and did not identified in Pakistan yet but chances are there to spread in Pakistan through Iran and currently Ug99 resistant wheat genotypes may become susceptible to this virulent. It is an unremitting straggle and should be continued in future to save wheat from rusts. Furthermore, rust resistant genes (Lr, Yr, Sr) as discovered world widely should be introduced in our wheat breeding program to add in new breeding lines through hybridization. Our results get support from the conclusions of Parveen et al., (2014); Kosgey et al., (2015); Bhardwaj et al., (2019); Gessese, (2019). However, Ejaz et al., (2012) found contrary results and reported insufficient stem resistance in Pakistani wheat genotypes. This might be due to study of different stem rust genes in different wheat genotypes.

Conclusion: The results of the study revealed that Pakistani wheat genotypes have enough resistance against stem rust (Sr) pathogens yet but satisfactory number of genes are not available for leaf rust ( $\mathrm{Lr}$ ) and stripe rust (Yr). Therefore, it is strongly proposed that local wheat germplasm should be strengthen through introduction of rusts resistant exotic genotypes developed by internationally to cope the future rust epidemics. Furthermore, in current climate change scenario, it is the need of hour to add in maximum discovered rusts resistance genes in newly developing candidate wheat lines to ensure food security in Pakistan.

Acknowledgements: The research project was conducted by the research fund provided by University of Sargodha under the program "Research and Development".

\section{REFERENCES}

Akhtar, N., A. Waseem, T. Mehmood, S. Bano, A. Raza and Ahsan Aziz (2018). Gene action appraisal for seed yield and related traits in bread wheat. The J. Anim. \& Plant Sci, 28: 1457-1465.

Basnet, B.R., P.R. Singh, S.A. Herrera-Foessel, A.M.H. Ibrahim, J. Huerta-Espino,V. Calvo-Salazar and J.C. Rudd. (2013). Genetic analysis of adult plant resistance to yellow rust and leaf rust in common spring wheat quaiu 3. P1. Dis. 97: 728-736.

Bhardwaj S. C. G.P. Singh., O, P. Gangwar., P, Prasad and S. Kumar. (2019). Status of Wheat Rust Research and Progress in Rust Management-Indian Context. Agron., 9:1-14.

Ejaz, M., M. Iqbal, A. Shahzad, A.I. Atiq-ur-Rehman and G. M. Ali. (2012). Genetic variation for markers linked to stem rust resistance genes in Pakistani wheat varieties. Crop Sci. 52: 2038-2648.

FAO. (2018). FAO statistical year book. Food and Agriculture organization (FAO) of the United Nations, Rome, Italy. Available at http://faostat3.fao.org/browse/Q/QC/E.

Gessese, M. K. (2019) Description of Wheat Rusts and Their Virulence Variations Determined through Annual Pathotype Surveys and Controlled MultiPathotype Tests. Advan. Agri., 2019: 1-7.

Government of Pakistan. (2019). Federal Bureau of Statistics. Economic Survey of Pakistan.

Jeffrey, G. E., S. L. Evans, S. Wolfgang and N.D. Peter. (2014). The past, present and future of breeding rust resistant wheat. Fron. P1. Sci. 5: 641-651.

Khan, M.A. (1997). Evaluation of Multiple Regression Models based on Epidemiological Factors to predict Leaf Rust on Wheat. Pakistan J. Agric. Sci. 34: 1-7. 
Kosgey, Z., J.O. Owuoche, M.A. Okiror and P.N. Njau. (2015). Inheritance of stem rust (Puccinia graminis Pers. F. Sp. Triticiericks and E. Hen) resistance in bread wheat (Triticum aestivum L.) lines to TTKST race. Int. J. Agron. Agric. Res.7: $1-13$.

McCallum, B.D., C.W. Hiebert, S. Cloutier, D. Bakkeren, S.B. Rosa, Humphreys DG, Marais GF, McCartney CA, Panwar V, Rampitsch C, Saville BJ, Wang X (2016). A review of wheat leaf rust research and the development of resistant cultivars in Canada. Canadian J P1 Path 38: 1-18.

Pal, D., S.C. Bhardwaj, D. Sharma, S. Kumari, M.V. Patial and P. Sharma. (2015). Assessment of Genetic Diversity and Validating Rust Resistance Gene Sources Using Molecular Markers in Wheat (Triticum aestivum L.). SABRAO J. Br. Genet. 47: 89-98.

Parveen, Z., M. Iqbal, S. Rahman, M. Younis, M. Nawaz, S.H. Raza and M.Z. Iqbal. (2014). Rust resistance evaluation of advanced wheat (Triticum aestivum L.) genotypes using PCR-based DNA markers. Pakistan J. Bot. 46: 251-257.

Pretorius, Z.A., Y. Jin, C.M. Bender, L. Herselman, and R. Prins. (2012). Seedling resistance to stem rust race $U g 99$ and marker analysis for $S r 2, S r 24$ and Sr31 in South African wheat cultivars and lines. Euphy. 186:15-23.
Qamar, M., S.D. Ahmad, M.S. Rabbani, Z.K. Shinwari and M. Iqbal. (2014). Determination of rust resistance genes in Pakistani bread wheats. Pakistan J. Bot. 46: 613-617.

Rahman, M., D. Hussain and Y. Zafar. (2002). Estimation of genetic divergence among elite cotton cultivars genotypes by DNA fingerprinting technology. Crop Sci. 42:2137-2144.

Singh, R.P.; Singh, P.K.; Rutkoski, J.; Hodson, D.P.; Xinyao He; Jorgensen, L.N.; Hovmoller, M.S. and Huerta-Espino (2016) Disease impact on wheat yield potential and prospects of genetic control. J. Annual Review of Phytopathology 54: 303-322.

Tadesse, W., S. Tawkaz, M.N. Inagaki, E. Picard and M. Baum. (2013). Methods and Applications of Doubled Haploid Technology in Wheat Breeding. ICARDA, Aleppo, Syria, pp 03.

Wang, H., F. Qin, Q. Liu, L. Ruan, R. Wang, Z. Ma, X. Li, P. Cheng and H. Wang. (2015). Identification and disease index inversion of wheat stripe rust and wheat leaf rust based on hyperspectral data at canopy level. J Spect ID 651810, 10 pages.

Yu, L.X., A. Morgounov, R. Wanyera, M. Keser, S.K.Singh and M. Sorrells. (2012). Identification of Ug99 stem rust resistance loci in winter wheat germplasm using genome-wide association analysis. Theo. Appl. Genet. 125: 749-758. 